\title{
De Sitter nonlinear sigma model and accelerating universe
}

\author{
Joohan Lee* \\ Department of Physics, University of Seoul, Seoul 130-743 Korea \\ Tae Hoon Le€† \\ Department of Physics and Institute of Natural Sciences, \\ Soongsil University, Seoul 156-743 Korea \\ Tae Yoon Moon $\ddagger$ and Phillial Ohई \\ Department of Physics and Institute of Basic Science, \\ Sungkyunkwan University, Suwon 440-746 Korea
}

(Dated: October 29, 2018)

\begin{abstract}
We consider a cosmology with a noncompact nonlinear sigma model. The target space is of de Sitter type and four scalar fields are introduced. The potential is absent but cosmological constant term $\Lambda$ is added. One of the scalar fields is time dependent and the remaining three fields have no time dependence but only spatial dependence. We show that a very simple ansatz for the scalar fields results in the accelerating universe with an exponential expansion at late times. It is pointed out that the presence of the energy density and pressure coming from the spatial variation of the three scalar fields plays an essential role in our analysis which includes $\Lambda=0$ as a special case and it discriminate from the standard $\Lambda$-dominated acceleration. We perform a stability analysis of the solutions and find that some solutions are classically stable and attractor. We also present a nonperturbative solution which asymptotically approaches an exponential acceleration and discuss possible cosmological implications in relation to dark energy. It turns out that the equation of state approaches asymptotically $\omega=-1$ both from above and below, but the crossing does not occur. It predicts present value of $\omega \sim-1 \mp 0.07$, which is within the region allowed by the observational data. This solution also exhibits a power law expansion at early times, and the energy density of the scalar fields mimics that of the stiff matter.
\end{abstract}

PACS numbers: 11.10.Lm, 95.36.+x, 98.80.-k

Keywords: nonlinear sigma model; cosmology; exponential acceleration; dark energy

\section{INTRODUCTION}

The recent cosmological observations [1] provide many precise data and arouse an explosion of recent interests in the cosmology. The most recent data and its cosmological interpretation [2] indicate that about $73 \%$ of our Universe is made of dark energy, the origin of which is one of the greatest puzzles in the modern cosmology [3].

It is highly conceivable that the dark energy is responsible for the late acceleration of the Universe [4] and many candidates have been proposed. The simplest approach for the accelerating universe is to introduce the cosmological constant 5, 6, 7, 8, 9] for the dark energy. Other approaches [4] include dynamical models of the cosmological constant [10, 11, 12, 13, 14, 15, 16, 17, 18, 19, 20, 21, 22, 23, 24]. Among them, most commonly proposed candidates are the quintessence, which is described by a scalar field minimally coupled to Einstein gravity with a potential [12, 15]. It is shown that the scalar energy density is subdominant in the matter dominated, and then, acceleration takes over at later stage of the cosmological evolution. Later the phantom model with a negative kinetic energy scalar field was proposed [25] to account for the region where the equation of state is less than $\omega=-1$, and quintom model where the ordinary scalar and the phantom are both introduced 26 to explain the crossing of the $\omega=-1$ line. In these models and subsequent works, various forms [11, 12, 13, 15, 17, 27] of the potential are introduced to account for the dark energy and the asymptotic acceleration is achieved through the quintessence or phantom dominance at late stage.

In this paper, we consider a cosmological model with a nonlinear sigma model [28] with a cosmological constant term. The target space is noncompact and is of four-dimensional de Sitter manifold and four scalar fields are introduced.

\footnotetext{
*Electronic address: joohan@kerr.uos.ac.kr

†Electronic address: thlee@ssu.ac.kr

${ }^{\ddagger}$ Electronic address: dpproject@skku.edu

$\S$ Electronic address: ploh@dirac.skku.ac.kr
} 
One of the motivations is that the scalar fields have a geometric origin and the potential term is not necessary. Another motivation is to consider the spatial dependence of the scalar fields and examine its consequences. To solve the Einstein equation, we assume that only one of the scalar fields is time dependent and the remaining three fields have no time dependence but only spatial dependence. We first show that a very simple ansatz can solve the Einstein equation which describes the acceleration of the universe with an exponential expansion at late stage. The spatial contribution in combination with the cosmological constant forms an effective cosmological constant and plays important roles in order to provide the necessary energy density and pressure. We will also show that the acceleration is possible even without the cosmological constant. It seems that this feature of contributing the energy density and pressure coming from the spatial variation of the scalar fields was not considered before in relation with dark energy.

Then, we perform a stability analysis of the solutions. We find that some of the solutions, depending on the values of the parameter given, are classically stable and attractor solutions. We will consider two cases where the target space has signature $(+,-,-,-)$ or $(-,+,+,+)$. Especially, in the $(+,-,-,-)$ case, the linear stability analysis fails, but we are able to find out that there exists a nonperturbative solution which asymptotically approaches the de Sitter acceleration, but at early times it is a power law expansion. In this case, the cosmological constant term is uniquely fixed in terms of the other parameter. These features differentiate the present analysis from the standard $\Lambda$-dominated late-time exponential acceleration.

One might think that adding a cosmological constant term with the scalar fields could be ad hoc, but it seems that at present, the dynamical models of the dark energy is not completely successful in solving the cosmological constant problem and many of them require some kind of fine tuning anyhow. Nevertheless, the aim of this paper is not to explain the smallness of the cosmological constant (the fine tuning problem in our approach is mentioned in the Conclusion and Discussion section), but to focus on the late-time exponential acceleration of the universe and stability of its behavior. It turns out that the accelerating universe requires some bound on the original cosmological constant term. In $(-,+,+,+)$ case, it require that the original cosmological constant term must be negative for stability, still the acceleration is possible and can be led by the scalar fields.

The paper is organized as follows. In Sec. II, we present noncompact nonlinear sigma model coupled with Einstein gravity with a cosmological constant term and discuss the ansatz which solves the equations in some generality. In Sec. III, we describe the exponential accelerating solution with de Sitter target space. In Sec. IV, the stability analysis is performed and allowed range of the cosmological constant is classified. In Sec. V, a nonperturbative solution is obtained and possible cosmological implications in relation to dark energy is given. Section VI includes the conclusion and discussion.

\section{THE ACTION AND COSMOLOGICAL CONSTANT}

We consider an action in which the Einstein gravity is coupled to a nonlinear sigma model with a cosmological constant term (in units of $M_{p}=1$ ):

$$
S=\int d^{4} x \sqrt{-g}\left[\frac{1}{2} R-\frac{g^{\mu \nu}}{\lambda^{2}} G_{\alpha \beta}(\Phi) \partial_{\mu} \Phi^{\alpha} \partial_{\nu} \Phi^{\beta}-\Lambda+\mathcal{L}_{\text {matter }}\right]
$$

where $\Phi^{\alpha}=\left(\phi, \sigma^{i}\right)(i=1,2,3), G_{\alpha \beta}$ is the metric of the noncompact target space, $\lambda^{2}$ is the self-coupling constant of the nonlinear sigma model and it is assumed to be positive. $\Lambda$ is the cosmological constant. The equations of motion are given by

$$
\begin{array}{r}
R_{\mu \nu}=\frac{2}{\lambda^{2}} G_{\alpha \beta} \partial_{\mu} \Phi^{\alpha} \partial_{\nu} \Phi^{\beta}+\Lambda g_{\mu \nu}+\hat{T}_{\mu \nu} \\
\frac{1}{\sqrt{-g}} \partial_{\mu}\left[\sqrt{-g} g^{\mu \nu} G_{\alpha \beta} \partial_{\nu} \Phi^{\beta}\right]=\frac{1}{2} \frac{\partial G_{\beta \gamma}}{\partial \Phi^{\alpha}} g^{\mu \nu} \partial_{\mu} \Phi^{\beta} \partial_{\nu} \Phi^{\gamma}
\end{array}
$$

where $\hat{T}_{\mu \nu}\left(=T_{\mu \nu}-g_{\mu \nu} T / 2\right)$ is assumed to take the perfect fluid form;

$$
T_{\nu}^{\mu}=\left(-\rho_{m}, p_{m}, p_{m}, p_{m}\right) .
$$

The matter sector satisfies the continuity equation; $\nabla_{\mu} T^{\mu \nu}=0$.

If we ignore the matter part, we can solve the Eqs. (2) and (3) with the following ansatz

$$
\phi=t, \quad \sigma^{i}=x^{i} .
$$


To check whether Eq. (2) can be solved (without $\left.\hat{T}_{\mu \nu}\right)$ with this ansatz, first note that if $\Lambda=0, g_{\mu \nu}\left(t, x^{i}\right)=+G_{\mu \nu}\left(\phi, \sigma^{i}\right)$ satisfies the equation as long as the scalar curvature of the space-time metric $g_{\mu \nu}$ and that of the target space $G_{\alpha \beta}$ are constants. Then, we can add an cosmological constant $\Lambda$ which has the same sign with these curvatures and the equation can still be satisfied [29, 30]. This has the effect of scaling the space-time metric via $g_{\mu \nu} \rightarrow(1+4 \Lambda / R) g_{\mu \nu}$, where $R$ is the scalar curvature constant. The cosmological constant could even have some value of the opposite sign with these curvatures as long as the absolute value of the cosmological constant is smaller than that of the target space, i.e., $|\Lambda|<|R| / 4$. This point can be extended further. Suppose $g_{\mu \nu}\left(t, x^{i}\right)=-G_{\mu \nu}\left(\phi, \sigma^{i}\right)$. Then, the scalar curvatures of the space-time and target space have opposite signature and the equation cannot be satisfied with $\Lambda=0$. But if we add $\Lambda$ such that the sign is the same as the space-time scalar curvature constant and the absolute value is greater than the scalar curvature of the target space, the equation can be satisfied. Also, one can check that the metric ansatz $g_{\mu \nu}(x)= \pm G_{\mu \nu}\left(\phi, \sigma^{i}\right)$ satisfies the Eq. (3). In summary, we find that the metric ansatz and (5) solve (21) and (3), and we have

$$
R_{\mu \nu}=\left( \pm \frac{2}{\lambda^{2}}+\Lambda\right) g_{\mu \nu}
$$

as long as the constant curvature condition is satisfied and without $\hat{T}_{\mu \nu}$.

The $\sigma^{i}=x^{i}$ ansatz first appeared in higher dimensional gravity theory in association with spontaneous compactification of the extra dimensions [29, 30]. It does not break the isotropy and homogeneity of the universe as long as we do not introduce the potential for the $\sigma$ fields. Also the $\phi=t$ has been exploited to unify early-time and late-time universe based on phantom cosmology [31]. Note that the quantity $\Lambda_{e f f} \equiv \pm 2 / \lambda^{2}+\Lambda$ plays the role of the effective cosmological constant and there is curvature constant restriction on the value of $\Lambda ; \pm 2 / \lambda^{2}+\Lambda$ must have the same signature as that of the space-time scalar curvature constant. The above aspect of the ansatz (5), (6) is quite a general feature of the nonlinear sigma model coupled to gravity. In this paper, we will consider the de sitter target space with

$$
G_{\alpha \beta}^{(\epsilon)}=\epsilon\left(1,-e^{2 \xi \phi},-e^{2 \xi \phi},-e^{2 \xi \phi}\right)
$$

with $\epsilon=\mp 1$ and $\xi$ being an arbitrary positive constant. It turns out that the spatial ansatz (5) provides contribution of the energy density and pressure such as to reveal diverse aspects of the late time exponential acceleration, not present in the standard cosmological constant dominated acceleration. We will also find that the allowed value of the cosmological constant divides further if required the stability.

\section{DE SITTER SOLUTION}

To discuss the cosmological implication of the solution (5), (6) with the de Sitter target space metric (7), we introduce the standard space-time metric via

$$
d s^{2}=-d t^{2}+a^{2}(t) d x_{i} d x^{i}
$$

With $H=\dot{a} / a$, the equation of motion (2) becomes

$$
\begin{aligned}
H^{2} & =\frac{2}{3 \lambda^{2}}\left[\epsilon\left(\frac{1}{2} \dot{\phi}^{2}-\frac{1}{2} e^{2 \xi \phi}{\dot{\sigma^{i}}}^{2}+\frac{1}{2 a^{2}}\left(\partial_{i} \phi\right)^{2}-\frac{1}{2 a^{2}} e^{2 \xi \phi}\left(\partial_{i} \sigma^{j}\right)^{2}\right)+\frac{\lambda^{2}}{2} \Lambda\right]+\frac{1}{3} \rho_{m} \\
\dot{H} & =-\frac{1}{\lambda^{2}} \epsilon\left[\dot{\phi}^{2}-e^{2 \xi \phi}{\dot{\sigma^{i}}}^{2}-\frac{1}{3 a^{2}}\left(\partial_{i} \phi\right)^{2}-\frac{1}{3 a^{2}} e^{2 \xi \phi}\left(\partial_{i} \sigma^{j}\right)^{2}\right]-\frac{1}{2}\left(1+\omega_{m}\right) \rho_{m}
\end{aligned}
$$

where $\omega_{m}=p_{m} / \rho_{m}$. The continuity equation implies $\rho_{m} \propto a^{-3\left(1+\omega_{m}\right)}$. Plugging the ansatz $\sigma^{i}=x^{i}$ and $\phi \equiv \phi(t)$ into the above equations, Eqs. (3), (9) and (10) become

$$
\begin{aligned}
0 & =\ddot{\phi}+3 H \dot{\phi}-3 \xi \frac{e^{2 \xi \phi}}{a^{2}}, \\
H^{2} & =\frac{2}{3 \lambda^{2}}\left[\epsilon\left(\frac{1}{2} \dot{\phi}^{2}-\frac{3}{2 a^{2}} e^{2 \xi \phi}\right)+\frac{\lambda^{2}}{2} \Lambda\right]+\frac{1}{3} \rho_{m} \\
\dot{H} & =-\frac{1}{\lambda^{2}} \epsilon\left[\dot{\phi}^{2}-\frac{1}{a^{2}} e^{2 \xi \phi}\right]-\frac{1}{2}\left(1+\omega_{m}\right) \rho_{m} .
\end{aligned}
$$

The second terms in both (12) and (13) are the contributions coming from the spatial variations of $\sigma_{i}$ which is essential for the subsequent analysis. 
The scalar dominance requires a check of whether the matter contribution term can be ignored at late times. Our solution corresponds to a linearly increasing scalar field with positive $\xi$ such that the kinetic energy terms and the second terms in both (12) and (13) are constant. Therefore, the contribution of the matter density which decreases as $a^{-3\left(1+\omega_{m}\right)}$ becomes negligible at late times and we ignore the matter part here after. Now, substitution of $\phi=t$ leads to

$$
a(t)=e^{\xi t}, \quad \xi=\sqrt{-\frac{2 \epsilon}{3 \lambda^{2}}+\frac{\Lambda}{3}}\left(\Lambda>\frac{2 \epsilon}{\lambda^{2}}\right) .
$$

In the above equation, we fixed the initial values by $a(0)=1$ and $\phi(0)=0$. Later, we will relax these initial conditions and accommodate more general conditions. This describes a de Sitter expansion of the universe. From here on, we will always assume $\sigma^{i}=x^{i}$ and study the time-dependent behavior of the Eqs. (11), (12), (13). Note that for the $\epsilon=+1$ case, the cosmological constant term has to be bigger than some positive value. In contrast, for the $\epsilon=-1$ case, it could be any value greater than a fixed negative value and we will see that stability adds further restrictions. It must especially be negative for stability; still the universe can accelerate and this is driven by the scalar fields. The cosmological constant term combines with the contribution from the scalar fields to form an effective cosmological constant $\Lambda_{\text {eff }}=3 \xi^{2}$ in Eq. (14).

\section{STABILITY}

To check the stability of the above solution, we first consider the following quantities,

$$
2 \xi \phi-2 N=X, \quad N=\ln (a)
$$

Plugging (15) into (11) (13), we obtain

$$
\begin{array}{r}
3 H^{2}+\dot{H}=-\frac{2 \epsilon}{\lambda^{2}} e^{X}+\Lambda \\
\ddot{X}+3 H \dot{X}-\left(6 \xi^{2}+\frac{4 \epsilon}{\lambda^{2}}\right) e^{X}+2 \Lambda=0
\end{array}
$$

The solution (14) corresponds to $X=0$ with $H=\xi, \dot{\phi}=1$. In order to accommodate more initial conditions, we consider the solution $X=X(0) \equiv \ln f$. Then, the effective cosmological constant becomes $\Lambda_{e f f}(f)=-2 \epsilon f / \lambda^{2}+\Lambda$, and the solution (14) is replaced by

$$
\phi=\sqrt{f} t+\phi(0), a(t)=a(0) e^{\sqrt{f} \xi t}, \quad \xi=\sqrt{-\frac{2 \epsilon}{3 \lambda^{2}}+\frac{\Lambda}{3 f}},
$$

with $2 \xi \phi(0)-2 \ln a(0)=\ln f, \Lambda>\frac{2 \epsilon f}{\lambda^{2}}$. Note that the exponent $\xi$ behaves under the change of the initial conditions when $\Lambda=0$ as follows; $f \rightarrow g, e^{\sqrt{f} \xi t} \rightarrow e^{\sqrt{g} \xi t}$.

The linear perturbation of Eq. (17) leads to

$$
\delta \ddot{X}+3 \sqrt{f} \xi \delta \dot{X}-\left(6 \xi^{2}+\frac{4 \epsilon}{\lambda^{2}}\right) f \delta X=0
$$

Introducing $\delta X \sim e^{\gamma t}$, Eq. (19) yields

$$
\gamma^{2}+3 \sqrt{f} \xi \gamma-\left(6 \xi^{2}+\frac{4 \epsilon}{\lambda^{2}}\right) f=0
$$

The solutions for the Eq. (20) are

$$
\begin{aligned}
\gamma_{+} / \sqrt{f} & =\frac{-3 \xi+\sqrt{33 \xi^{2}+\frac{16 \epsilon}{\lambda^{2}}}}{2} \\
\gamma_{-} / \sqrt{f} & =\frac{-3 \xi-\sqrt{33 \xi^{2}+\frac{16 \epsilon}{\lambda^{2}}}}{2}
\end{aligned}
$$




\begin{tabular}{|c|c|c|c|c|}
\hline \hline Name & $\epsilon$ & $\xi$ & Stability & $\Lambda$ \\
\hline \hline$(\mathrm{A})$ & +1 & $\xi>0$ & $\gamma_{+}>0, \gamma_{-}<0$, Unstable, saddle point & $\Lambda>2 f / \lambda^{2}$ \\
\hline$(\mathrm{B})$ & -1 & $16 / 33 \lambda^{2} \leq \xi^{2}<2 / 3 \lambda^{2}$ & $\gamma_{ \pm}<0$, Stable, attractor & $-6 f / 11 \lambda^{2} \leq \Lambda<0$ \\
\hline$(\mathrm{C})$ & -1 & $\xi^{2}>2 / 3 \lambda^{2}$ & $\gamma_{+}>0, \gamma_{-}<0$, Unstable, saddle point & $\Lambda>0$ \\
\hline$(\mathrm{D})$ & -1 & $\xi^{2}<16 / 33 \lambda^{2}$ & $\gamma_{ \pm}$, Imaginary, stable, attractor & $-2 f / \lambda^{2}<\Lambda<-6 f / 11 \lambda^{2}$ \\
\hline$(\mathrm{E})$ & -1 & $\xi^{2}=2 / 3 \lambda^{2}$ & $\gamma_{+}=0, \gamma_{-}<0$ & $\Lambda=0$ \\
\hline$(\mathrm{F})$ & +1 & $\xi^{2}=1 / 3 \lambda^{2}$ & Nonperturbative & $\Lambda=3 f / \lambda^{2}$ \\
& & $\phi=\sqrt{f} t+\phi_{0}+A \ln \left(1+C e^{-\sqrt{f} \gamma t}\right)$ & \\
\hline
\end{tabular}

TABLE I: Various accelerating solutions and their stability

From these equations, we have the following cases:

(A). For the $\epsilon=+1$ case, $\gamma_{+}>0$ and $\gamma_{-}<0$ for any $\xi$. Hence, the solution is unstable. It corresponds to a saddle point. The cosmological constant has to be positive.

For the $\epsilon=-1$ case, there are three cases. In all three cases, the cosmological constant has to be negative for stability, but the late acceleration is achieved by the scalar fields.

(B). In the case $\frac{16}{33 \lambda^{2}} \leq \xi^{2}<\frac{2}{3 \lambda^{2}}, \gamma_{ \pm}$are both negative and the solution is stable and an attractor. For $\xi^{2}=\frac{16}{33 \lambda^{2}}$, the root is degenerate with $\gamma_{+} / \sqrt{f}=\gamma_{-} / \sqrt{f}=-3 \xi / 2$.

(C). For $\xi^{2}>\frac{2}{3 \lambda^{2}}, \gamma_{+}>0$ and $\gamma_{-}<0$, so the solution is unstable and corresponds to a saddle point.

(D). In the case $\xi^{2}<\frac{16}{33 \lambda^{2}}, \gamma_{ \pm}$becomes imaginary and the perturbation is oscillatory and it is an attractor.

The linear perturbation can be integrated explicitly. For (A), (B) and (C), Eq. (19) yields

$$
\begin{aligned}
& \delta X=A e^{\gamma_{+} t}+B e^{\gamma_{-} t} \\
& \delta H=\frac{-2 \epsilon f}{\lambda^{2}}\left(\frac{A e^{\gamma_{+} t}}{\gamma_{+}+6 \sqrt{f} \xi}+\frac{B e^{\gamma_{-} t}}{\gamma_{-}+6 \sqrt{f} \xi}-C_{0} e^{-6 \sqrt{f} \xi t}\right),
\end{aligned}
$$

where $C_{0}=A /\left(\gamma_{+}+6 \sqrt{f} \xi\right)+B /\left(\gamma_{-}+6 \sqrt{f} \xi\right)$. Note that when $\epsilon=+1$, the second term in Eq. (24) diverges for the value $\xi^{2}=1 / 3 \lambda^{2}$. This might imply that linear perturbation fails in this case and in fact, there exist a nonperturbative solution as will be discussed in the next section. For case (D), we have

$$
\delta X=D \cos \left(\omega t+\theta_{0}\right) e^{-\frac{3}{2} \sqrt{f} \xi t}
$$

where $\omega^{2}=\frac{f}{4}\left(\frac{16}{\lambda^{2}}-33 \xi^{2}\right)$ and

$$
\delta H=-E e^{-6 \sqrt{f} \xi t}+F(t) e^{-\frac{3}{2} \sqrt{f} \xi t}
$$

where

$$
\begin{aligned}
E & =36 D f \sqrt{f} \xi \cos \theta_{0} / \lambda^{2}\left(81 f \xi^{2}+4 \omega^{2}\right)+8 D f \omega \sin \theta_{0} / \lambda^{2}\left(81 f \xi^{2}+4 \omega^{2}\right), \\
F(t) & =36 D f \sqrt{f} \xi \cos \left(\omega t+\theta_{0}\right) / \lambda^{2}\left(81 f \xi^{2}+4 \omega^{2}\right)+8 D f \omega \sin \left(\omega t+\theta_{0}\right) / \lambda^{2}\left(81 f \xi^{2}+4 \omega^{2}\right) .
\end{aligned}
$$

We comment on the case $\xi^{2}=2 / 3 \lambda^{2}$ separately. It corresponds to when the cosmological constant $\Lambda$ is zero. In this case, one of the roots $\gamma_{+}$of Eq. (21) becomes zero, and the other root $\gamma_{-}$is negative. Its stability is indecisive at this level. These solutions, their stability and contents of the cosmological constant are summarized in Table I. It is interesting to note that in the $\epsilon=-1$ case, any value greater than a fixed negative of the cosmological constant was allowed in (14), but stability requires its upper bound must be zero.

\section{NONPERTURBATIVE SOLUTION}

It turns out that in the $\epsilon=+1$ case, an explicit nonperturbative solution can be found. To see that, let us first assume $e^{\xi \phi}=\sqrt{f} a$. Then, Eq. (12) suggests that for nontrivial solution, we must have

$$
H^{2}=\frac{1}{3 \lambda^{2}} \dot{\phi}^{2}, \quad \lambda^{2} \Lambda=3 f
$$


The first of the above equation yields $\xi^{2}=1 / 3 \lambda^{2}$. This value of $\xi^{2}$ was the one where linear perturbation failed in the previous section. Substituting the ansatz $a=\frac{1}{\sqrt{f}} e^{\xi \phi}$ into (11), we obtain

$$
\ddot{\phi}+3 \xi \dot{\phi}^{2}-3 f \xi=0
$$

Note that this equation describes particle motion where constant external force and velocity square dependent frictional force are acting. When $\dot{\phi}(0)^{2}<f$, the constant force term dictates the particle motion at early times and it accelerates until the velocity reaches the terminal velocity $\dot{\phi}(\infty)=\sqrt{f}$. When $\dot{\phi}(0)^{2}>f$, the friction term dominates at early times and it decelerates until the velocity reaches the terminal velocity $\dot{\phi}(\infty)=\sqrt{f}$.

We can find the solution for the above Eq. (29) as follows

$$
\phi(t)=\sqrt{f} t+\phi(0)-\frac{1}{3 \xi} \ln (1+C)+\frac{1}{3 \xi} \ln \left(1+C e^{-6 \sqrt{f} \xi t}\right),
$$

with $\xi=\sqrt{\frac{1}{3 \lambda^{2}}}$ and

$$
a(t)=a(0) e^{\sqrt{f} \xi t}\left(\frac{1+C e^{-6 \sqrt{f} \xi t}}{1+C}\right)^{\frac{1}{3}} .
$$

The constant $\mathrm{C}$ remains arbitrary as long as the validity of the solution is confined within the region $1+C e^{-\sqrt{f}} \gamma t>0$. Note that the solution (30) has two arbitrary integration constants and it is a complete solution for the ansatz $e^{\xi \phi}=\sqrt{f} a$ with $\phi(0)$ and $\dot{\phi}(0)=\sqrt{f}(1-2 C) /(1+C)$. It indicates that starting with an arbitrary value of $\mathrm{C}$, the solution converges rapidly to $\phi(t)=\sqrt{f} t$ and $a(t)=e^{\sqrt{f} \xi t}$. There is a wide range of initial conditions in which the solution rapidly converges to de Sitter acceleration.

To see the solution more closely, let us divide the case with $C>0$ and $C<0$. First, note that the condition $1+C e^{-\sqrt{f} \gamma t}>0$ puts a restriction on the range of $|C|$, i.e., $|C|<1$ when $C<0$. When $C>0$, it could be arbitrary. We introduce a time scale defined by $|C|=e^{-6 \sqrt{f} \xi t_{*}}$. Then, Eq. (30) can be written as

$$
\phi(t)= \begin{cases}\frac{1}{3 \xi} \ln \left(\sinh \left(3 \sqrt{f} \xi\left(t+t_{*}\right)\right)\right)+\tilde{\phi}(0), & \left(C<0,0<t_{*}<\infty\right) \\ \frac{1}{3 \xi} \ln \left(\cosh \left(3 \sqrt{f} \xi\left(t+t_{*}\right)\right)\right)+\tilde{\phi}(0), & \left(C>0, \quad-\infty<t_{*}<\infty\right)\end{cases}
$$

where $\tilde{\phi}(0)=\phi(0)-\ln \left(\sinh \left(3 \sqrt{f} \xi t_{*}\right)\right) / 3 \xi$ for $C<0$ and $\tilde{\phi}(0)=\phi(0)-\ln \left(\cosh \left(3 \sqrt{f} \xi t_{*}\right)\right) / 3 \xi$ for $C>0$ in $(32)$. And we also have

$$
a(t)= \begin{cases}\tilde{a}(0)\left(\sinh \left(3 \sqrt{f} \xi\left(t+t_{*}\right)\right)\right)^{\frac{1}{3}}, & \left(C<0,0<t_{*}<\infty\right) \\ \tilde{a}(0)\left(\cosh \left(3 \sqrt{f} \xi\left(t+t_{*}\right)\right)\right)^{\frac{1}{3}}, & \left(C>0,-\infty<t_{*}<\infty\right)\end{cases}
$$

where $\tilde{a}(0)=a(0) /\left(\sinh \left(3 \sqrt{f} \xi t_{*}\right)\right)^{\frac{1}{3}}$ for $C<0$ and $\tilde{a}(0)=a(0) /\left(\cosh \left(3 \sqrt{f} \xi t_{*}\right)\right)^{\frac{1}{3}}$ for $C>0$ in (33). Note that $\left.\dot{\phi}\right|_{t=-t_{*}}$ becomes singular in (32), but our initial time is chosen to be 0 , and it is outside the range of dynamics. Had we chosen our initial time to be $t_{i}$, the singularity would occur at $t=t_{i}-t_{*}$. We will assume that $|C| \sim 1$ so that $t_{*}$ is nearly the initial time.

Let us assume that the initial time $t=0$ is chosen when the universe is still at the matter-dominated epoch and examine the early-time behavior of the solutions (32) and (33). Then, for $C<0$ with Eqs (32) and (33), we have

$$
\phi(t) \sim \frac{1}{3 \xi} \ln \left(t+t_{*}\right)+\tilde{\phi}_{0}, a(t) \sim\left(t+t_{*}\right)^{\frac{1}{3}} .
$$

The logarithmic time-dependent $\phi$ field [11, 12, 16, 17] also appears in the quintessence with exponential potential. The energy density $\rho_{\phi}=\frac{1}{\lambda^{2}} \dot{\phi}^{2} \sim\left(t+t_{*}\right)^{-2} \sim a^{-6}$ scales the same as the stiff matter density and is known as a scaling solution. In our case, the scaling behavior holds only at early times, and as time goes by, the full solution (32) will take over. For $C>0$ with Eqs. (32) and (33), we see that the $\phi$ field and the scale factor $a(t)$ remains constant up to first order in time, and as time goes by, both quantities begins to grow. There is no scaling behavior in this case. Note that for both cases, $\Omega_{\phi}=\frac{\rho_{\phi}}{\rho_{c}}=1$ due to the first condition of Eq. (28). This is because in the above solution, matter contribution was neglected. It would be interesting to check whether the dominance of the energy density of scalar fields emerges from the matter-dominated epoch when the matter contributions are included. 
Let us discuss some issues of dark energy with this solution. First, we have the acceleration given by

$$
\frac{\ddot{a}}{a}=f \xi^{2} \frac{1 \pm 10 e^{-6 \sqrt{f} \xi\left(t+t_{*}\right)}+e^{-12 \sqrt{f} \xi\left(t+t_{*}\right)}}{\left(1 \pm e^{-6 \sqrt{f} \xi\left(t+t_{*}\right)}\right)^{2}}
$$

where + in the numerator is for $C>0$ and - is for $C<0$. We see that for $C>0$, it is always accelerating. For $C<0$, there is a transition time where the scale factor changes from deceleration to acceleration. It is given with $t_{*} \sim 0$ by

$$
t_{t r}=\frac{1}{6 \sqrt{f} \xi} \ln (5+2 \sqrt{6}) \sim 0.48 t_{0} .
$$

where $t_{0}$ is the current age of the universe [32]. This fix $\sqrt{f} \xi \sim 0.79 / t_{0}$. Next, equation of state with this value of $\sqrt{f} \xi$ is given by

$$
\omega=-1 \mp 8 \frac{e^{-6 \sqrt{f} \xi\left(t+t_{*}\right)}}{\left(1 \mp e^{-6 \sqrt{f} \xi\left(t+t_{*}\right)}\right)^{2}} \sim-1 \mp 0.07 .
$$

For $C>0$ with an upper sign in (37), $\omega$ approaches -1 from below. $\omega$ is singular at $t=-t_{*}$ and again it is outside the range of dynamics. For $C<0$, it approaches -1 from above. And there is no singularity. In both cases, there is no crossing the $\omega=-1$, but asymptotically approaches the $\omega=-1$ line. We find that this number for the equation of state in (37) is in the region allowed by the observational data [2], but inclusion of matter might change the result somewhat.

\section{CONCLUSION AND DISCUSSION}

We presented a de Sitter nonlinear sigma model coupled to Einstein gravity in order to describe the current acceleration of the Universe. It has some characteristic features as follows. Out of the four scalar fields, only one of them is time-dependent and the remaining three fields have only spatial dependence. If the time dependent scalar field is phantom, then the remaining fields are ordinary scalar fields, or vice versa. The formal case could also be thought of as the dilatonic phantom coupled with triplet of scalar fields, whereas the latter case as the dilaton coupled with triplet of phantom scalar fields. Since kinetic energy of both positive and negative sign exists, it could be thought of as a quintom model [26] with dilaton interaction between the two sectors. But the quintom model only considers time-dependent fields. A specific form of the potential is not needed to achieve the late-time exponential acceleration, but introduction of a potential could produce subdominant behavior of the scalar fields as in the quintessence. It is suspected that the potential does not modify the late-time exponential behavior, because it does not change the Eq. (13).

We find that a simple ansatz provides the constant energy density and results in an accelerating universe with an exponential expansion. The balance between the pressures coming from the time-dependent field and spatialdependent fields makes it possible to achieve the exponential acceleration. It is pointed out that the target space of Euclidean de Sitter space with signature $(+,+,+,+)$ cannot produce such balance and exponential acceleration of the universe. The model has essentially two parameters, $\xi$ and the cosmological constant term $\Lambda$. $\sqrt{f} \xi M_{p}$ plays the role of the Hubble constant and is a function of the strength of the self-coupling constant and the cosmological constant term $\Lambda$. Consider, for example, the nonperturbative case with $\xi^{2}=1 / 3 \lambda^{2}$ and $\Lambda=3 f / \lambda^{2}$. Recall $f=e^{2 \xi \phi(0) / M_{p}} / a^{2}(0)$ and let us assume $a(0) \sim 1$. We mention a couple of cases where $\sqrt{f} \xi M_{p} \sim 10^{-61} M_{p}$ and $\Lambda \sim 10^{-122} M_{p}^{2}$ can be realized. In the first case with $\phi(0) \sim-M_{p}$ and $\lambda \sim 1 / 250$, we have $\xi \phi(0) \sim-145 M_{p}$. If the scale when the nonlinear sigma model sets in is of the order of Gev with $\phi(0) \sim-1 \mathrm{GeV}$, this requires extremely weak coupling constant with $\lambda \sim 10^{-21}$. In this case, we have $\xi \phi(0) \sim-190 M_{p}$. These are fine tunings which can yield the small Hubble constant and the cosmological constant.

Stability analysis shows some of the solutions, depending on the values of the parameter $\xi$, are classically stable and attractor solutions. They require that the original cosmological constant term must be negative, still the acceleration is possible led by the scalar fields. In one case, where the cosmological constant term is uniquely fixed, there is a nonperturbative solution which asymptotically approaches the de Sitter phase of acceleration. This solution also exhibits a power law expansion at early times, and the energy density of the scalar fields mimics the matter energy density. It remains to be seen whether the stability survives when the analysis is extended to spatial variations.

The present analysis indicates that the acceleration phase can be dominated by the nonlinear sigma model. We only focused on the late time behavior except the nonperturbative case. To show whether this behavior of scalar 
dominance can emerge from matter-dominated epoch, the analysis has to be extended including the contribution of matter density at early times which was neglected. Finally, whether the de Sitter nonlinear sigma model could come from particle physics as an effective low energy field theory remains to be seen. These aspects needs further investigation.

Note added: After the completion of this work, we became aware of Ref. [33] where the ansatz (5) each multiplied by some constant factors to have Minkowski background also appeared in the cosmological context of the Lorentz violating massive graviton models [34]. These models deal with flat background metric. However, in our de Sitter background solution, a linear perturbation of the metric in the action (11) does not result in any massive graviton mode even though the ansatz (5) spontaneously break the diffeomorphism invariance. This can be readily seen by checking that the mass term which is of the second order in the perturbations cancels out in the second term of the action in (11). Moreover, the de Sitter background solution allows the modification where each of the ansatz in (5) can be multiplied by a same constant factor only which still does not yield Lorentz violating mass term. Perhaps, it could be possible to generate a mass term by a suitable deformation of the target space metric in the action (11), which is beyond the scope of this work, but nevertheless whose implications would be worthwhile to be explored in detail.

\section{ACKNOWLEDGEMENT}

We thank the anonymous referee for pointing out Ref. [33] to us. We also thank S. T. Hong for early participation and Y.-Y. Keum for useful information on dark energy. The work of THL was supported by the Korea Research Foundation Grant funded by the Korean Government (MOEHRD, Basic Research Promotion Fund) (KRF-2007313-C00165). The work of PO is supported by the Science Research Center Program of the Korea Science and Engineering Foundation through the Center for Quantum Spacetime(CQUeST) of Sogang University with Grant No. R11-2005-021.

\section{REFERENCES}

[1] S.J. Perlmutter et al., Astrophys. J. 517, 565 (1999); A.G. Riess et al., Astrophys. J. 659, 98 (2007), astro-ph/0611572 C. L. Bennett et al., Astrophys. J. Suppl. 148, 1 (2003); D. J. Eisenstein et al., Astrophys. J. 633, 560 (2005); D.N. Spergel et al. (WMAP Collaboration), Astrophys. J. Suppl. 170, 377 (2007), astro-ph/0603449

[2] E. Komatsu et al. (WMAP Collaboration), Astrophys. J. Suppl. 180, 330 (2009).

[3] A.R. Liddle and D.H. Lyth, Cosmological Inflation and Large-Scale Structure (Cambridge University Press, Cambridge, 2000).

[4] See S.M. Carroll, AIP Conf. Proc. 743, 16 (2004), astro-ph/0310342, E. J. Copeland, M. Sami and S. Tsujikawa, Int. J. Mod. Phys. D15, 1753 (2006); V. Sahni and A. Starobinsky, Int. J. Mod. Phys. D15, 2105 (2006), and references therein.

[5] S. Weinberg, Rev. Mod. Phys. 61, 1 (1989).

[6] S. M. Carroll, W. H. Press, and Turner, E. L., Annu. Rev. Astron. Astrophys. 30, 499 (1992).

[7] V. Sahni and A. Starobinsky, Int. J. Mod. Phys. D9, 373 (2000), astro-ph/9904398

[8] T. Padmanabhan, Phys. Rept. 380, 235 (2003); Curr. Sci. 88, 1057 (2005).

[9] P. J. E. Peebles and B. Ratra, Rev. Mod. Phys. 75, 559 (2003).

[10] L. H. Ford, Phys. Rev. D35, 2339 (1987).

[11] C. Wetterich, Nucl. Phys. B302, 668 (1988).

[12] B. Ratra and P. J. E. Peebles, Phys. Rev. D37, 3406 (1988).

[13] P. J. E. Peebles and B. Ratra, Astrophys. J. 325, L17 (1988).

[14] Y. Fujii and T. Nishioka, Phys. Rev. D42, 361 (1990).

[15] R. R. Caldwell, R. Dave and P. J. Steinhardt, Phys. Rev. Lett. 80, 1582 (1998).

[16] E. J. Copeland, A. R. Liddle and D. Wands, Phys. Rev. D57, 4686 (1998).

[17] P. G. Ferreira and M. Joyce, Phys. Rev. D58, 023503 (1998).

[18] I. Zlatev, L. Wang and P. J. Steinhardt, Phys. Rev. Lett. 82, 896 (1999).

[19] P. J. Steinhardt, L. Wang and I. Zlatev, Phys. Rev. D59, 123504 (1999).

[20] J. E. Lidsey, D. Wands and E. J. Copeland, Phys. Rep. 337, 343 (2000).

[21] T. Chiba, T. Okabe and M. Yamaguchi, Phys. Rev. D62, 023511 (2000).

[22] C. Armendariz-Picon, V. F. Mukhanov and P. J. Steinhardt, Phys. Rev. Lett. 85, 4438 (2000); Phys. Rev. D63, 103510 (2001). 
[23] M. Malquarti, E. J. Copeland, A. R. Liddle and M. Trodden, Phys. Rev. D67, 123503 (2003).

[24] T. Chiba and R. Takahashi, Phys. Rev. D75, 101301 (R) (2007).

[25] R. R. Caldwell, Phys. Lett. B545, 23 (2002).

[26] B. Feng, X. Wang, X. Zhang, Phys. Lett. B607, 35 (2005); Z.-K. Guo, Y. S. Piao, X. M. Zhang and Y. Z. Zhang, Phys. Lett. B608, 177 (2005); B. Feng, M. Li, Y.-.S Piao and Y. X. Zhang, Phys. Lett. B634, 101 (2006).

[27] J. A. Frieman, C. T. Hill, A. Stebbins and I. Waga, Phys. Rev. Lett. 75, 2077 (1995); L. P. Chimento and A. S. Jakubi, Int. J. Mod. Phys. D5, 71 (1996); Ph. Brax and J. Martin, Phys. Lett. B468, 40 (1999); Phys. Rev. D61, 103502 (2000); V. Sahni and L. Wang, Phys. Rev. D62, 103517 (2000); A. Albrecht and C. Skordis, Phys. Rev. Lett. 84, 2076 (2000); T. Barreiro, E. J. Copeland and N. J. Nunes, Phys. Rev. D61, 127301 (2000); L. A. Urena-Lopez and T. Matos, Phys. Rev. D62, 081302(R) (2000); S. M. Carroll, M. Hoffman and M. Trodden, Phys. Rev. D68, 023509 (2003); S. Lee, K. A. Olive and M. Pospelov, Phys. Rev. D70, 083503 (2004).

[28] S. V. Ketov, Quantum Non-linear Sigma Models (Springer, Berlin, 2000).

[29] C. Omero and R. Percacci, Nucl. Phys. B165, 351 (1980).

[30] M. Gell-Mann and B. Zwiebach, Nucl. Phys. B260, 569 (1985); Phys. Lett. B147, 111 (1984); Phys. Lett. B141, 333 (1984).

[31] S. Nojiri and S. D. Odintsov, Gen. Rel. Grav. 38, 1285 (2006).

[32] E. E. O. Ishida, R. R. R. Reis, A. V. Toribio and I. Waga, Astropart. Phys. 28,547 (2008).

[33] S. L. Dubovsky, P. G. Tinyakov and I. I. Tkachev, Phys. Rev. D72, 084011 (2005).

[34] N. Arkani-Hamed, H. Georgi and M. D. Schwartz, Annals Phys. 305, 96 (2003); N. Arkani-Hamed, H.-C. Cheng, M. A. Luty and S. Mukohyama, JHEP 0405, 074 (2004); V. A. Rubakov, hep-th/0407104 S. L. Dubovsky, JHEP 0410,076 (2004); S. L. Dubovsky, P. G. Tinyakov and I. I. Tkachev, Phys. Rev. Lett. 94, 181102 (2005); G. 't Hooft, arXiv:0708.3184. 